\title{
IMPACTO DEL PROCESO EDUCACIONAL EN LA CARRERA DE INGENIERÍA AGROFORESTAL, URACCAN LAS MINAS
}

\author{
Letisia Castillo Gómez ${ }^{[1]}$ \\ Bismarck Lee León ${ }^{[2]}$ \\ Maribel Duriéz González ${ }^{[3]}$
}

\section{Resumen}

El objetivo de esta investigación fue valorar el impacto de la formación recibida en la Universidad de las Regiones Autónomas de la Costa Caribe Nicaragüense, Recinto Las Minas en el desarrollo personal y profesional de quienes se graduaron en Ingeniería Agroforestal, cohorte 1998-2002.

Se desarrolló en el escenario de la vida de estos profesionales que se ubicaron geográficamente en los Municipios del triángulo minero (Siuna, Rosita y Bonanza), perteneciente a la Región Autónoma del Atlántico Norte (RAAN), mediante el paradigma cualitativo y el interaccionismo simbólico como enfoque teórico metodológico. Se realizaron entrevistas narrativas individual y grupal, así como análisis documental.

Los hallazgos destacan que las relaciones interpersonales, la cultura institucional y comunitaria en que se formaron los agroforestales, les permitieron la adopción de valores y la obtención de nuevos hábitos intelectuales y morales, que están explícitamente declarados en el currículo de la carrera y en los ejes transversales de la universidad, pero estos carecen de un marco conceptual y metodológico. Además, se garantizó mediante el proceso de enseñanza-aprendizaje la construcción de aprendizajes significativos, habilidades y actitudes directamente relacionadas con la profesión y su ejercicio, en correspondencia con las características del contexto, apoyado en las prácticas y el conocimiento endógeno de la Región. Lo que les ha propiciado efectos positivos en el ámbito personal y profesional, con mejores ingresos económicos, reconocimiento social, calidad de vida y mejor persona.

Palabras claves: Formación profesional, currículo, desarrollo personal y profesional, seguimiento a graduados, beneficios.

\section{Introducción}

Las políticas educativas actualmente se encaminan a la acreditación de instituciones y/o a programas de estudio, esto implica cumplir una serie de estándares de calidad

[1] MSc. Docencia Universitaria. Secretaria Académica URACCAN Las Minas

[2] MSc. Docencia Universitaria. Vicerrector URACCAN Las Minas

[3] PhD. Tutora. Docente maestría Docencia Universitaria. mduriez@cablenet.com.ni 
aplicados a diferentes factores sujetos de evaluación, entre los cuales están los profesionales. En este marco, es necesario darle seguimiento y valorar el impacto de la formación transcurrido un tiempo después.

Con base en el manual de funcionamiento de URACCAN (2007) la egresada/ egresado forma parte de la comunidad y es, en cierto modo, vivo testigo de la gestión universitaria. Por ello, la URACCAN se propone mantener una estrecha vinculación con los graduados, de modo que puedan aportar su experiencia como estudiantes y como profesionales inmersos en la vida social, donde deben distinguirse por su identificación con los fines de la Universidad, su cosmovisión indígena y afrodescendiente, su respeto a la interculturalidad, su testimonio ético y su compromiso con la Autonomía Regional.

El seguimiento de los profesionales graduados y particularmente la valoración del impacto ha sido un reto insoslayable. Ésta es una carencia detectada desde el proceso de autoevaluación y evaluación externa llevados a cabo en el año 2004, donde se expresa literalmente que: No existen mecanismos de seguimiento a los graduados en la universidad, el cual permita el establecimiento de programas de capacitación, especialización y ubicación laboral de estos/estas. No exceptuando de esta problemática al Recinto Universitario Las Minas y especialmente a los de la carrera de Ingeniería Agroforestal.

La carrera de Ingeniería Agroforestal es una de las carreras cuya oferta no ha variado. En el año 2007 se efectúa el primer estudio de seguimiento a graduados/graduadas, donde se obtiene que esta carrera, es la que mejor aceptación tiene por parte de la sociedad del triángulo minero, manifestándose en la cantidad de estudiantes matriculados cada año de acuerdo con los registros académicos oficiales.

\section{Revisión de literatura}

La educación en este trabajo se concibe como un proceso de formación integral del ser social, que supone una dinámica del sujeto educando con otros sujetos y con su entorno, fomentando el manejo de destrezas, habilidades, hábitos, actitudes, valores éticos y morales, indispensables en la vida presente y futura; con la finalidad de promover su pleno desarrollo desde sus modos de ser, vivir y estar en el mundo.

Hooker (2008) plantea:

Las universidades deben contribuir a que los pueblos originarios y comunidades afrodescendientes puedan ejercer sus derechos a practicar y revitalizar sus tradiciones y costumbres culturales, lo que implica el derecho a revitalizar, utilizar, desarrollar y transmitir a las generaciones futuras sus historias, idiomas, tradiciones orales, 
filosofías, sistemas de escritura y literaturas, debiendo el Estado proporcionar los medios necesarios para tal cometido (p.9).

Consecuencias de las prácticas pedagógicas monoétnicas, no se ha logrado visibilizar las prácticas tradicionales de enseñanza-aprendizaje de los y las indígenas, los negros/negras, los campesinos/campesinas, ni han permitido que sus propias propuestas educativas sean reconocidas ni implementadas, fuera de los ámbitos cotidianos.

Está ampliamente documentado, que los pueblos indígenas y comunidades étnicas tienen idiomas, patrimonio, culturas y conocimientos ricos y diversos, adquiridos de sus relaciones entre sí y sus ecosistemas, que se reproducen de generación en generación y que se manifiestan a través de sus tradiciones, creencias, costumbres y ceremonias. Esos conocimientos, han sido incorporados a sus prácticas, para tener ingreso a otros conocimientos, transmitirlos y compartirlo (ONU, 1999).

Por consiguiente se indican que la perspectiva pedagógica de la Educación Superior está en estado potencial, debido a la complejidad de las demandas de la sociedad actual y sobre todo en contextos multiculturales donde es necesario incorporar el diálogo de saberes y el reconocimiento de la diversidad.

La URACCAN es una institución académica formadora de profesionales y técnicos mediante la implementación de una instrucción científica y una educación generadora e integral, que reconoce los derechos políticos, económicos, sociales y culturales de los pueblos indígenas, afrodescendientes y comunidades étnicas de la Costa Caribe nicaragüense, que enriquece su identidad étnica y respeta los caracteres específicos de sus culturas, garantizando de esta manera la igualdad en la diversidad, la unidad nacional y la integridad territorial de la nación, elementos necesarios para impulsar el desarrollo y consolidar el proceso de autonomía y su transformación económica, política y social.

Los efectos o impactos de la formación recibida deben ser evaluados. Según Fernández y García (s/f), la evaluación de impactos puede considerarse un caso particular de los procesos de evaluación de la calidad, sobre los que no abundan las conceptuaciones ni sus implicaciones metodológicas, por lo que es necesario introducir algunas ideas acerca de la concepción de este proceso. Responde a su lógica general, sin embargo, busca esencialmente la medición de aquellas variables asociadas a los impactos que son objeto de atención en la evaluación, es decir, las que ponen de manifiesto el logro de las transformaciones esenciales propuestas en la mejora proyectada (p.1).

Por lo que en este trabajo se asume que la evaluación de impacto es "un proceso orientado a medir los resultados generados (cambios y causas) por las acciones formativas desarrolladas en el escenario socio profesional originado de las mismas, al 
cabo del tiempo" (Ferrández, 2006). Buscan el efecto que tuvo la formación en la vida de los y las formados/formadas (p.20).

Para Jaramillo et al. (2002), la calidad de la formación recibida repercute en una mejor calidad de vida, en la posibilidad de tomar decisiones más acertadas, de alcanzar un estatus personal más alto, incrementa sus oportunidades de educación permanente, aumenta las expectativas de vida, y un estilo de vida más saludable.

Campos (2002), considera que la educación es el mejor medio para inculcar hábitos de prudencia y superación, por lo que se puede afirmar sin escrúpulos que el fin de toda instrucción intelectual para la masa del pueblo debe consistir en cultivar el sentido común y prepararla para formar un juicio práctico verdadero de las circunstancias que lo rodean.

Así mismo, Martínez (2006) plantea que enseñar y aprender contenidos curriculares de aprendizajes profundos y relevantes para las diferentes titulaciones que comparten contenidos de interés social y ético, equivale a promover la integración del impacto social y ético en los mismos.

\section{Materiales y método}

El paradigma en que se basa esta investigación es cualitativo con el enfoque teórico metodológico del Interaccionismo Simbólico que sirvió para determinar los significados sociales compartidos que tienen los profesionales graduados de la carrera de Ingeniería Agroforestal sobre el impacto de la formación académica recibida en su desarrollo personal y profesional.

El universo lo constituyeron 32 graduados/graduadas de la carrera de Ingeniería Agroforestal que estudiaron con el plan de estudio 1998-2002. De los cuales, 21 son del sexo masculino y 11 femeninos, pertenecientes a la etnia mestiza. Geográficamente se encuentran ubicados en los municipios de Siuna (12), Rosita (7), Bonanza (6), Waslala (2), Río Blanco (1), Juigalpa (1), Managua (1) y en el país Estados Unidos de Norteamérica (2).

En el momento de la investigación sólo 4 mujeres se encontraban desempleadas las cuales se localizan en el municipio de Siuna. Los 28 restantes trabajan en instituciones como: el Instituto Nacional Forestal (INAFOR), Instituto Nacional de Transferencia Agropecuaria (INTA), Instituto Nacional Tecnológico (INATEC), Ministerio Agropecuario y Forestal (MAGFOR), Universidad de las Regiones Autónomas de la Costa Caribe Nicaragüense (URACCAN), Bluefields and Indian Caribean University (BICU), Alcaldías Municipales específicamente en las Unidades de Gestión Ambiental y Caja Rural Nacional (CARUNA). 
La muestra seleccionada fue de 20 agroforestales de la cohorte generacional 19982002, a través del muestreo de casos homogéneos.

Los métodos empleados fueron: el análisis documental, la entrevista y la discusión grupal. Las técnicas utilizadas fueron: análisis documental, entrevista narrativa individual y entrevista grupal.

\section{Resultados y discusión}

\section{Desarrollo personal de los profesionales graduados de Ingeniería Agroforestal después de la formación recibida en URACCAN Las Minas}

La URACCAN a través de su programa de formación de Ingeniería Agroforestal ofrece un escenario donde las oportunidades para el cambio son sustanciales en sus estudiantes. No es posible hablar de educación sin suponer la presencia de una orientación de valores, ya sea de manera voluntaria o involuntaria. Lo que coincide con Gómez (2005) que los valores forman parte de cualquier organización y por lo tanto su transmisión es inherente a la actividad cotidiana de cualquier institución educativa.

De acuerdo con la revisión documental realizada, se evidenció que el currículo de la carrera 1998-2002, integra los valores al aprendizaje de manera intencionada y consciente, aunque estos no están conceptualizados en el mismo. Están declarados y enmarcados en la formación de los ingenieros y las ingenieras. Por lo que están en los fines, el perfil de egreso y el diseño de la realización del proceso de formación en cuanto al sistema de conocimientos, habilidades y actitudes que permiten una especialización en su saber técnico, y al mismo tiempo una formación humanista.

Los valores de género e interculturalidad no se destacan en el currículo de una manera explícita, han constituidos ejes transversales en el desarrollo curricular, los que son muy pertinentes para el contexto sociocultural donde se encuentra la universidad. Coincidiendo con Martínez (1995) que estos temas transversales:

son contenidos culturales relevantes y valiosos, necesarios para la vida y la convivencia, que figuran de manera especial el modelo de ciudadano que demanda cada sociedad, a través de una educación en valores que permita a los estudiantes sensibilizarse y posicionarse ante los problemas, enjuiciarlos críticamente y actuar con un compromiso libremente asumido.

Es necesario resaltar que en las entrevistas a los agroforestales, externaron que a su ingreso a esta universidad traían consigo temores, inseguridades, prejuicios estereotipados hacia determinados grupos étnicos, en este caso, al pueblo mayangna, además de mucha dependencia emocional. 
Como resultado de la implementación del currículo de la carrera, de las relaciones interpersonales, de la cultura institucional y comunitaria, los profesionales graduados sienten que son más tolerantes, independientes, responsables, honestos, respetuosos de la diversidad, equitativos, solidarios, defensores de los derechos individuales y colectivos, apropiados de la Autonomía, seguros de quien son y orgullosos de su profesión y de su universidad.

No obstante, cabe señalar, que todos los valores expresados anteriormente están carentes de conceptos en el currículo de la carrera, lo que evidencia que son las prácticas diarias las que construyen estas definiciones. Esto tiene una doble lectura, según Hawes (2003), por un lado, una cierta ambigüedad conceptual proveniente ya sea de la falta de claridad o de la incapacidad de lograr consensos significativos entorno a los mismos y la segunda lectura es que el tema de los valores está abierto, correspondiendo a la comunidad académica la construcción de sus significaciones en la dinámica diaria.

Además de la adopción de valores como base para el desarrollo personal han obtenido nuevos hábitos intelectuales y morales para cada una de las áreas de su vida, clave para su crecimiento personal. Entre los que se destacan el hábito de estudio, la puntualidad, la transmisión de conocimientos, energía positiva para actuar, ayudar a otros a desarrollarse, compartir parte de sus experiencias, respetar la diversidad, promover la Autonomía como un valor social, los que también se explicitan en el currículo de la carrera.

Por lo que se evidencia un cambio en la manera de pensar y actuar de los ingenieros e ingenieras después de terminar la universidad, que les ha proporcionado un crecimiento como persona, lo que coincide con el planteamiento de Campos (2002), citando a Stuart Mill (1909) al considerar que la educación es el mejor medio para inculcar hábitos de prudencia y superación, por lo que se puede afirmar sin escrúpulos que el fin de toda instrucción intelectual para la masa del pueblo debe consistir en cultivar el sentido común y prepararla para formar un juicio práctico verdadero de las circunstancias que lo rodean.

\section{Desarrollo profesional de los graduados y graduadas de Ingeniería Agroforestal después de la formación recibida en URACCAN Las Minas}

La organización del currículo de Ingeniería Agroforestal 1998-2002, se ajusta a lo que se denomina modelo tradicional o basado en la estructura de contenidos. Lo que coincide con uno de los modelos curriculares de Hawes y Donoso (2003), currículo basado en la estructura de los contenidos. El que tiene tres características fundamentales:

- El o la estudiante ingresa a la carrera y sólo puede egresar de la misma. 


\section{CIENCIAS SOCIALES}

- El plan de estudio está compuesto por unidades denominadas "asignaturas" cada una representa un campo de conocimientos determinado.

- La formación se inicia con cursos de "ciencias básicas", pasando por cursos disciplinarios, hasta cursos orientados a asignaturas profesionalizantes específicas.

El mismo, acentúa que los ingenieros y las ingenieras agroforestales, son personas con una formación integral, capacitados para desarrollar sistemas alternativos de producción basados en el uso racional y sostenible de los recursos naturales propios de cada localidad, así como en la recuperación o incorporación de áreas manejadas deficientemente y altamente degradadas por las prácticas del monocultivo, y orientado hacia la búsqueda de un entendimiento entre la sociedad y la naturaleza según el currículo de Ingeniería Agroforestal.

Los graduados manifestaron que haber egresado por la universidad en la carrera de Ingeniería Agroforestal les permitió tener un avance en el desarrollo de conocimientos más especializados en los temas agroforestales, desarrollo de habilidades, actitudes y experiencias en el área profesional así como un mayor nivel de reflexión y análisis. Lo que se manifiesta en quienes ya incursionaron en el campo laboral.

Las graduadas desempleadas expresaron que aunque no están trabajando en estos momentos, se sienten seguras de lo aprendido y que no temen poner en práctica los conocimientos y las habilidades obtenidas en la universidad.

La combinación de la teoría con la práctica de los diferentes cursos, no aparece metodológicamente orientado en el mismo currículo, por lo que se infiere que es parte de las estrategias metodológicas de cada docente en el desarrollo de las disciplinas. Lo que trae consigo que la práctica no sea una disposición metodológica de la formación desde el currículo y queda en mano de la labor docente su desarrollo.

En el desarrollo curricular solo se pudo profundizar los conocimientos en las áreas forestal y social, no siendo así en cultivo y ganadería, específicamente en los temas de plagas y enfermedades. Además, las prácticas de campo fueron muy pocas, considerando la importancia de la combinación teoría-práctica para la formación profesional.

Las graduadas desempleadas expresaron que se necesita más prácticas en el campo, porque es ahí donde se van a desempeñar y solicitan que se profundice más en los rubros de cultivo y ganadería.

Esta situación pone al descubierto un problema en la implementación del currículo 1998-2002, el cual plantea el desarrollo de los componentes agrícola, forestal y pecuario en un sistema y que en la ejecución se hizo necesario la profundización en las áreas de ganadería, agricultura y prácticas de campo. 


\section{Efectos del proceso educacional en el desarrollo personal y profesional}

El proceso de formación recibido mediante la carrera de Ingeniería Agroforestal (currículo 1998-2002), trajo consigo considerables efectos en el desarrollo personal y profesional. Los que fueron determinados por los ingenieros y las ingenieras independientemente de su empleabilidad como beneficios personales, sociológicos y científico-tecnológicos.

Dentro de los beneficios personales destacaron: incremento de capacidades, desarrollo de nuevas habilidades, aumento de la productividad individual, mejores relaciones personales, adopción de nuevos valores, obtención de hábitos, el aprendizaje de nuevas cosas, mejor calidad de vida y mejor persona.

Por lo anterior se deduce, que la formación recibida tiene efectos positivos en el plano personal de los graduados y las graduadas, independientemente del estado de empleabilidad que han tenido después de titularse. Lo que se visualiza en la realización personal de los sujetos, oportunidades formativas, crecimiento personal, independencia, satisfacción de las propias expectativas de vida, desarrollo intelectual, seguridad social, ocupación y productividad.

En el orden sociológico se distinguieron beneficios como: estatus personal y respetabilidad social por la consideración que se otorga a las personas provistas de un título académico determinado, además de la empleabilidad incrementada, siendo tradicional que una personal con un título profesional tiene mayores posibilidades de obtener empleo que alguien sin el mismo.

El hecho de estar titulados es un factor importante del por qué están insertos en el mercado laboral o se desarrollan personalmente, además del sentido personal que le atribuyen a la ubicación laboral en su proyecto de vida y al efecto social que significa el que se realicen en su elección de manera responsable.

Lo que concuerda con la Teoría del Capital Humano expuesta por Aguilar (2009), en el sentido de que cuando un individuo es altamente educado desarrolla competencia y/o habilidades que lo hacen más productivo, el aumento en su productividad se ve reflejado en unos ingresos mucho mayores en comparación con aquél que no posee estas habilidades.

Los beneficios de orden científico-tecnológico que resaltaron fueron: el desarrollo de conocimientos, valores, habilidades y actitudes para poder adquirir, construir y transferir conocimientos en beneficio de la sociedad y la ampliación de las bases científicas de las tecnologías propias de la profesión. 


\section{CIENCIAS SOCIALES}

Por lo antes expresado se infiere que los graduados y las graduadas adquirieron los conocimientos técnicos para desarrollarse profesionalmente que aportan al desarrollo de las comunidades a través de soluciones a problemas enmarcados en su profesión y fomentan de una manera responsable el espíritu de formación y superación.

Lo que coincide con Martínez (2006) que una universidad de calidad y de servicio público es aquella que hace más digna la sociedad, convirtiendo a sus estudiantes tanto en excelentes profesionales, como en ciudadanos y ciudadanas cada vez más cultos y críticos.

Al mismo tiempo los graduados y graduadas consideran la necesidad de actualización por medio de programas de educación continua, debido a los acelerados avances científico-técnicos.

Lo que refuerza Jaramillo, et al (2002) que para los individuos la calidad de la formación recibida repercute en una mejor calidad de vida, en las posibilidades de tomar decisiones más acertadas, de alcanzar un estatus personal más alto, incrementa sus oportunidades de educación permanente, aumenta las expectativas de vida y un estilo de vida más saludable.

\section{Conclusiones}

La formación recibida en la URACCAN Las Minas tuvo un impacto positivo en los profesionales de Ingeniería Agroforestal, cohorte 1998-2002, lo que les ha permitido tener un desarrollo personal y profesional.

La implementación del currículo de Ingeniería Agroforestal 1998-2002, las relaciones interpersonales, la cultura institucional y comunitaria en que se formaron los graduados y las graduadas les permitió la adopción de valores como base para el desarrollo personal y la obtención de nuevos hábitos intelectuales y morales para cada una de las áreas de su vida, clave para su crecimiento personal.

El currículo de la carrera tiene explícitamente declarado los valores éticos de formación y existen temas transversales como género e interculturalidad que constituyen ejes vertebradores del trabajo académico; pero estos carecen de un marco conceptual y metodológico, por lo que son las acciones, palabras sentimientos y reacciones de quienes están inmersos en el proceso, que construyen dichas definiciones.

Tanto el currículo como el desarrollo del mismo garantizaron un proceso de enseñanza-aprendizaje orientada a la construcción de aprendizajes significativos, habilidades y actitudes directamente relacionadas con la profesión y su ejercicio en correspondencia a las características del contexto y apoyado en las prácticas y saberes endógenos. 
En la implementación del currículo se exteriorizan fortalezas de los componentes forestal y social y debilidades en cuanto al conocimiento y desarrollo de pecuaria y cultivo. Aunque en la práctica profesional no se reconoce como un obstáculo para el desarrollo profesional por las actitudes de permanente superación adquiridas en la universidad.

El proceso educacional propició efectos positivos en los agroforestales en el ámbito personal y profesional. Lo que se refleja de manera general en mejores ingresos económicos, reconocimiento social, calidad de vida y mejor persona. Los fueron determinados por los ingenieros y las ingenieras como beneficios personales, sociológicos y científico-tecnológicos.

Existe la necesidad del desarrollo de programas de educación continua a fin de actualizar periódicamente a los graduados, ante el acelerado avance de las ciencias y las tecnologías.

\section{Lista de referencias}

Aikman, S. (1995). Los pueblos indígenas y sus territorios. Perspectivas vol. XXV, No. 4.

Campos, R. G. (2002).Tesis doctoral. La Empleabilidad de las perspectivas de la economía moderna. [En línea] disponible en: http://www.eumed.net/tesis/gcr/cap2.doc [2009 28 de Agosto].

Fernández, F.A. y García, G. A. (s/f). Evaluación de la calidad de la maestría en Ciencias de la Educación: una propuesta en condiciones de amplio ingreso. Ponencia para el Sexto Congreso Internacional de la Educación Superior Universidad 2008. Habana, Cuba.

Ferrández L. E. (2006). La evaluación de impacto en el Máster de Formación de Formadores. CIFO-FLC. Informe de investigación. Universidad Autónoma de Barcelona, Departamento de Pedagogía Aplicada. España.

Freire, P. (1987). Pedagogía del oprimido. 17a ed. Río de Janeiro: Paz e Terra. Brasil.

Gómez, L.F. (2005). El impacto de la universidad en la formación de los alumnos. Instituto Tecnológico y de Estudios Superiores de Occidente, México.

Hawes, G. (2003). Un currículum para la formación profesional en la universidad. Talca. Universidad de Talca. Instituto de Investigación y desarrollo educacional. Proyecto Mecesup. 
Hawes, G. y Donoso, S. (2003). Currículum universitario. Características, construcción, instalación. Talca. Universidad de Talca. Instituto de Investigación y desarrollo educacional. Proyecto Mecesup.

Hooker, A. (2008). Educación superior y ciencia y tecnología en América Latina y el Caribe: Respuestas frente a la expansión y a la diversificación. Ponencia I Congreso Nacional de Educación Superior en Nicaragua.

Jaramillo, A., Ortiz, J., Zuluaga, E., Almonacid, P. y Acevedo, S. (2002). Los egresados y la calidad de la universidad EAFIT. Universidad EAFIT, oficina de planeación integral. Medellin, Colombia.

Martínez, M. J. (1995). Los temas transversales. Buenos Aires: Magisterio del Río de la Plata. Argentina.

Martínez, M. (2006). Formación para la ciudadanía y Educación Superior. Revista Iberoamericana de Educación No 42. Universidad de Barcelona, España.

Puhiera, A. (2006). Los retos de la universidad en la educación intercultural. Dossier Pedagogía Universitaria. Maestría en Docencia Universitaria. Universidad de las Regiones Autónomas de la Costa Caribe Nicaragüense. RAAN, Nicaragua.

Tunnerman, C. (1996). La Educación Superior en el umbral del Siglo XXI, UNESCO CRESALC, Caracas.

URACCAN (2007). Manual de funcionamiento y organización institucional. Reforma del presentado y aprobado al CUU en el 2005. Universidad de las Regiones Autónomas de la Costa Caribe Nicaragüense. Nicaragua. 